Balcazar, A. (2018). Gobernanza corporativa, una propuesta para el mejoramiento en la gestión administrativa y financiera en el hospital E.S.E Nuestra Señora del Carmen Tabio - Cundinamarca. Contaduría Universidad de Antioquia, 73, 13-32.

Doi: https://doi.org/10.17533/udea.rc.n73a01

\title{
Gobernanza corporativa, una propuesta para el mejoramiento en la gestión administrativa y financiera en el hospital E.S.E Nuestra Señora del Carmen Tabio - Cundinamarca
}

Ana María Balcazar Daza

anacoris2@hotmail.com

Universidad de Cundinamarca 

Gobernanza corporativa, una propuesta para el mejoramiento en la gestión administrativa y financiera en el
hospital E.S.E nuestra señora del Carmen Tabio-Cundinamarca

Resumen: En el departamento de Cundinamarca se ha cuestionado la gestión administrativa y financiera dentro de los hospitales, los cuales, de acuerdo con los resultados de las auditorías emitidas por la Contraloría de Cundinamarca, en gran mayoría, no muestran resultados administrativos y financieros de forma positiva. El objetivo de esta investigación es verificar la gestión administrativa y financiera del Hospital E.S.E Nuestra Señora del Carmen, Tabio-Cundinamarca y establecer que los resultados negativos de esta gestión se deben al problema de Riesgo Moral y Asimetría de la Información que plantea la Teoría de Agencia. Para este fin, se efectuará una revisión del informe de auditoría emitido por la contraloría de Cundinamarca con el fin de probar el anterior argumento. A posteriori se verificará la viabilidad de presentar una propuesta para el hospital de acuerdo con los planteamientos de la Gobernanza Corporativa. Para alcanzar dicho fin, se efectúa una revisión de documentos y artículos, con el fin de demostrar que es viable generar una propuesta de mejoramiento administrativo y financiero, de acuerdo con lo expuesto por dicha teoría. Se concluye que los problemas de gestión administrativa y financiera del Hospital E.S.E Nuestra Señora del Carmen, Tabio-Cundinamarca se deben a problemas de Riesgo Moral, Selección Adversa y por Asimetrías de la Información, para lo cual es viable proponer un mejoramiento en la gestión administrativa y financiera de acuerdo con lo planteado por la Gobernanza Corporativa.

Palabras clave: Teoría de agencia, gobernanza corporativa, gestión administrativa, gestión financiera.

Corporate governance: a proposal for improving the administrative and financial management of the E.S.E Nuestra Señora del Carmen Hospital, Tabio-Cundinamarca

Abstract: The Department of Cundinamarca has been concerned about the administrative and financial management within hospitals, which, for the most part, do not show positive results, according to the results of the auditing issued by the Cundinamarca Comptroller's Office. The aim of this research is to verify the administrative and financial management of the E.S.E Nuestra Señora del Carmen Hospital, Tabio-Cundinamarca, and establish that the negative results in this management are due to the problems of Moral Risk and Information Asymmetry posited by the Agency Theory. To this end, a review of the auditing report issued by the Cundinamarca Comptroller's Office will be conducted with the aim of proving the above argument. The viability of presenting a proposal for the hospital according to the propositions from Corporate Governance will be verified a posteriori. To reach this aim, a review of documents and articles is conducted, in order to demonstrate that it is viable to generate a proposal of administrative and financial management improvement, as stated by said theory. It is concluded that the problems of the administrative and financial management of the E.S.E Nuestra Señora del Carmen Hospital, Tabio-Cundinamarca, are due to problems of Moral Risk, Adverse Selection and Information Asymmetries, for which it is viable to propose an administrative and financial management improvement as stated by Corporate Governance.

Keywords: Agency Theory, Corporate Governance, administrative management, financial management.

La gouvernance corporative, une proposition pour améliorer la gestion administrative et financière à l'hôpital E.S.E. Nuestra Señora del Carmen, à Tabio, Cundinamarca en Colombie

Résumé: dans le département de Cundinamarca, la gestion administrative et financière des hôpitaux a été questionnée car, d'après les résultats des audits menés par la Contraloría (organisme de contrôle) de Cundinamarca, la plupart d'entre eux ne montrent pas des résultats administratifs et financiers positifs. L'objet de cette recherche est de vérifier la gestion administrative et financière de l'E.S.E. Hospital Nuestra Señora de Tabio Cundinamarca afin d'établir que les résultats négatifs de cette gestion sont dus au problème du Risque Moral et d'Asymétrie d'Information proposé par la Théorie de l'Agence. Pour ce faire, nous mènerons une révision du rapport d'audit fait par l'organisme de contrôle de Cundinamarca afin de démontrer cet argument. A posteriori, nous vérifierons la viabilité de faire une proposition à l'hôpital basée sur les lignes directrices de la Gouvernance Corporative. À cette fin, nous ferons une révision de documents et d'articles pour démontrer la viabilité de créer une proposition d'amélioration administrative et financière d'après la Théorie de l'Agence. Nous parviendrons à conclure que les problèmes de gestion administrative et financière de l'E.S.E. Hospital Nuestra Señora de Tabio Cundinamarca sont causés par le Risque Moral, la Sélection Défavorable et l'Asymétrie d'Information. C'est pour cela qu'il est viable de proposer une amélioration à la gestion administrative et financière basée sur la Gouvernance Corporative.

Mots-clés : théorie de l'agence, gouvernance corporative, gestion administrative, gestion financière.

Governança corporativa, uma proposta para o melhoramento na gestão administrativa e financeira no hospital “E.S.E. Nuestra Señora del Carmen” em Tabio - Cundinamarca

Resumo: No departamento de Cundinamarca tem se questionado a gestão administrativa e financeira dentro dos hospitais, os quais, segundo os resultados das auditorias emitidas pela "Contraloría de Cundinamarca" (que é a instituição encarregada do controle fiscal desse departamento), na sua maioria, não apresentam resultados administrativos e financeiros positivos. O objetivo desta pesquisa é verificar a gestão administrativa e financeira do Hospital "E.S.E. Nuestra Señora del Carmen" de Tabio Cundinamarca e estabelecer que os resultados negativos dessa gestão são devidos ao problema de Risco Moral e Assimetia da informação que supõe o Dilema da Agência (ou Problema do principal-agente). Com este intuito se realizará uma revisão do relatório de auditoria emitido pela "Contraloría de Cundinamarca" a fim de comprovar o anterior argumento. Posteriormente se verificará a viabilidade de apresentar uma proposta para o Hospital conforme aos fundamentos da Governança Corporativa. Para atingir tal objetivo, se fará uma revisão de documentos e artigos a fim de demonstrar que é viável gerar uma proposta de melhora administrativa e financeira, conforme o argumentado por essa teoria. Conclui-se que os problemas de gestão administrativa e financeira do Hospital "E.S.E Nuestra Señora del Carmen" de Tabio Cundinamarca são devidos a problemas de Risco Moral, Seleção Adversa e por Assimetrias da informação, pelo que é viável sugerir uma melhora na gestão administrativa e financeira de acordo com os fundamentos da Governança Corporativa.

Palavras Chave: Dilema da Agência, Governança Corporativa, gestão administrativa, gestão financeira. 
Cont. udea (julio-diciembre), pp. 13-32. (C) Universidad de Antioquia-2018.

\title{
Gobernanza corporativa, una propuesta para el mejoramiento en la gestión administrativa y financiera en el hospital E.S.E Nuestra Señora del Carmen Tabio - Cundinamarca
}

\author{
Ana María Balcazar Daza \\ Doi: https://doi.org/10.17533/udea.rc.n73a01
}

Primera versión recibida en agosto de 2018 - Versión final aceptada en septiembre de 2018

\section{Introducción}

$\mathrm{D}$ e acuerdo con los informes de auditoría emitidos por la contraloría de Cundinamarca, el sector hospitalario en este departamento ha mostrado deficiencias en su gestión administrativa y financiera. En consecuencia, es necesario replantear la metodología en cuanto la gestión interna de este sector organizacional; la cual está sumergida en diversas reglamentaciones públicas.

La gobernanza al interior de estas instituciones podría constituirse como un factor decisivo dentro del mejoramiento de los indicadores administrativos y financieros, en la medida que permitiría identificar dificultades dentro de la administración, y de esta manera, generar las acciones correctivas respectivas.

En consecuencia, el objetivo de esta investigación es verificar la gestión administrativa y financiera del Hospital E.S.E Nuestra Señora del Carmen de Tabio Cundinamarca y demostrar que los resultados negativos de esta gestión se deben al problema de Riesgo Moral, Selección Adversa y Asimetría de la Información los cuales son planteados por la Teoría de Agencia. Para este fin, se efectuará una revisión del informe de auditoría de la contraloría de Cundinamarca con el fin de probar el anterior argumento.

Posteriormente, se verificará la viabilidad de presentar una propuesta para el hospital de acuerdo con lo planteado por la Gobernanza Corporativa.

Con este propósito, se efectúo una revisión de artículos publicados en revistas indexadas, para sustentar el planteamiento del mejoramiento de la gestión administrativa y financiera, mediante la incorporación de la Gobernanza Corporativa en la gestión organizacional; y evidenciar una propuesta en 
Balcazar, A. Gobernanza corporativa, una propuesta para el mejoramiento en la gestión administrativa...

contribución a la consecución de indicadores administrativos y financieros positivos, dentro de la organización objeto de estudio, disminuyendo impactos por selección adversa (proceso que nace como consecuencia de la asimetría de la información, en el cual por desinformación se ofrece lo peor a un tercero) y riesgo moral (es la consecuencia que asumen terceros, por decisiones egoístas en beneficio propio, por parte de los directivos dentro de la organización).

Es por ello necesario establecer controles (gobernanza corporativa) con el fin de incrementar los niveles de productividad a través de incentivos (aumentos salariales, reconocimientos, privilegios, estabilidad) encaminados a promover la lealtad de los empleados a todo nivel dentro de la organización. Aunque esto genera un aumento en los costos dentro del hospitales, la relación costo beneficio a largo plazo sería favorable por la disminución de los costos de transacción originados por problemas de riesgo moral y asimetrías de la información, que deterioran la estructura financiera de la entidad.

De acuerdo con la estructura del texto, se expondrán inicialmente los postulados de la Teoría de Agencia y Gobernanza Corporativa. A posteriori, se mostrará un cuadro que evidencia la gestión administrativa y financiera del Hospital E.S.E Nuestra Señora del Carmen de Tabio Cundinamarca, después se mostrará el resultado de la revisión de los casos de éxito en gestión administrativa y financiera en algunas instituciones a nivel mundial tras la implementación del modelo de Gobernanza Corporativa, y finalmente se mostrará la propuesta para el hospital.

Como conclusión de la investigación, se puede establecer que los problemas de la gestión administrativa y financiera dentro del Hospital E.S.E Nuestra Señora del Carmen de Tabio se deben a los problemas expuestos por la Teoría de Agencia, los cuales son Riesgo Moral y Selección adversa como consecuencia de la Asimetría de la Información.

De acuerdo con lo encontrado dentro de la revisión de los artículos y textos se puede concluir, además, que existe una relación directa entre la incorporación de los postulados de la Gobernanza Corporativa, en el mejoramiento administrativo y financiero de las organizaciones que los aplican. En consecuencia, es acertado generar una propuesta al sector hospitalario de Cundinamarca de acuerdo con lo planteado por la Teoría de Gobernanza Corporativa.

\section{Marco Teórico}

\section{Teoría de la Agencia.}

La TA o enfoque contractual, conocida también como enfoque "Principal-Agente". Según los profesores norteamericanos Michel Jensen y William Meckling (1976), quienes definen la relación de agencia como un contrato bajo cuyas cláusulas una o más personas (Principal) contrata a otra persona (Agente) para realizar determinado 
servicio en su nombre, lo que implica delegación de autoridad. (Escobar, y Benavides, y Perafán, H 2016, p.207)

Esta teoría está enmarcada en el conflicto de intereses que se presenta en la relación, entre principal (dueño) y agente (directivos) dentro de la organización, por lo cual es necesario crear incentivos que logren evitar los conflictos de intereses presentados entre los principales y los agentes.

La Teoría de Agencia expone dos problemas a encontrar dentro de las organizaciones, el Riesgo Moral y la Selección Adversa como consecuencia de la Asimetría de la Información.

El Riesgo Moral, se presenta cuando los directivos dentro de las organizaciones tienen intereses personales que interfieren con los objetivos de los dueños de las empresas, y en consecuencia sus decisiones están orientadas a satisfacer las necesidades personales en detrimento de los objetivos corporativos.

Es por ello necesario establecer controles, con el fin de incrementar los niveles de productividad a través de incentivos (aumentos salariales, reconocimientos, privilegios, estabilidad) encaminados a promover la lealtad de los empleados a todo nivel dentro de la organización. Aunque esto implicaría un aumento en los costos dentro de la organización, la relación costo beneficio a largo plazo sería favorable por la disminución de estos Riesgos Morales, que implican altos costos operativos (transacción). De igual forma, es necesario empoderar a los administradores, con el fin de identificarlos con la organización y de esta forma lograr mayores rentabilidades financieras.

Así pues, este enfoque (servidor) no se centra en las motivaciones del administrador de los recursos, sino en las formas de facilitar y mejorar las estructuras de poder, lo cual asegura que el ambiente laboral del gestor lo incentive a adoptar un comportamiento en pro de la organización y favorecer que el gestor ostente el doble cargo de pertenecer a la junta directiva y ser el administrador, pues implicará mejoras en la efectividad y por tanto, mayores retornos para los accionistas. (Donaldson, 1990; Donaldson y Davis, 1991; Muth y Donaldson, 1998) citado por (Escobar, y Benavides, y Perafán, H 2016, p.206)

Surge esta teoría que busca tener una mirada más sociológica y psicológica de este individuo, en la que el agente es confiable pues basa su toma de decisiones en los intereses de la compañía a la que sirve, en vista de que está comprometido con el éxito de esta (Davis, Schoorman y Donaldson, 1997; Donaldson, 1990; Donaldson y Davis, 1991; Hassan, Marimuthu y Johl, 2015).

Otro de los problemas que enfrentan las organizaciones de acuerdo con la Teoría de Agencia consiste en las diferencias en cuanto a la información que poseen cada uno de los agentes económicos, lo cual se denomina Asimetría en la Información. Como consecuencia de la Asimetría de la Información, surge el concepto de Selección Adversa, el cual evidencia que se pueden 
Balcazar, A. Gobernanza corporativa, una propuesta para el mejoramiento en la gestión administrativa...

presentar fallas en el proceso de la toma de decisiones, pese a las diferencias en cuanto al manejo de la información por parte de los agentes involucrados. La Selección Adversa evidencia que, a causa de la falta de información, no se dará un intercambio justo. (Akerlof, 1970; Holmstrom, 1979; Stiglitz y Weiss, 1981; Demsetz, 1983; Usategui, 1999; Menéndez, 2001; Arévalo y Ojeda, 2004; Montoya y Montoya, 2005; Gómez, 2008; Ganga y Burotto, 2009; Manasliski y Varela, 2009; Briceño, 2010; Guzmán y Trujillo, 2011; Ganga y Burotto, 2012)

A continuación, se exalta los aportes que han efectuado algunos autores frentes a la Teoría de Agencia. Según Oman, Fries y Buiter (2003) la Teoría de la Agencia puede direccionarse para las empresas públicas y privadas, las cuales como organizaciones cuentan con normas, leyes, reglamentos, es así como el propósito de la gestión empresarial se fundamenta en:

Facilitar y estimular el desempeño de las empresas mediante la creación y el mantenimiento de los incentivos que motivan a la información corporativa para maximizar la eficiencia operativa de las empresas, el rendimiento de los activos y el crecimiento de la productividad a largo plazo. - Limitar a los gestores el abuso de poder sobre los recursos corporativos — si dicho abuso hace que se ocasione liquidación de activos o desvío de recursos de la empresa para su uso privado y/o causa desperdicio importante de recursos controlados por las corporaciones (el llamado 'problema de agencia') - que son otra manera en que se puede manifestar la conducta egoísta del gestor. - Proporcionar los medios para supervisar el comportamiento del gerente, garantizar la responsabilidad corporativa y establecer una protección razonable costo-efectiva de los intereses de los inversores y la sociedad de cara a la información corporativa”.) Citado por (Escobar, y Benavides, y Perafán, H 2016, p.218)

Lefort (2003), enfoca la Teoría de la Agencia, en el concepto de Asimetría de la Información (diferencias en la información en los diferentes agentes), lo cual trae como consecuencia la Selección Adversa (Fallas en las decisiones y en el intercambio a causa de la Asimetría de la Información). Según Lefort, se debe garantizar en las relaciones empresariales un intercambio justo, mediante el suministro de la misma información a todos los actores dentro de la organización.

Para González y Hernández (2006) Siempre existirán diferencias de intereses entre los dueños de las organizaciones y los administradores de las mismas, por lo tanto, es necesario de estipular controles y reglas claras con el fin de minimizar el conflicto entre estos actores en la organización. Los controles son tanto internos como externos. En los controles externos se encuentra el entorno legal y la regulatorio, y en los controles internos se encuentran las políticas y reglamentaciones internas.

Jensen y Meckling (1976), Shleifer y Vishny, reafirman lo expuesto por González y Hernández (2006), en el cual afirman que las organizaciones necesitan de estructuras de Gobierno al interior, con el fin de controlar y 
supervisar a los gestores, cuyos intereses no deben distar de los intereses de los propietarios.

Para Gruszczynski (2006), la gobernanza de agencia reduce al mínimo los costos que se derivan de los conflictos entre propietarios y gerentes de la organización, a través de la supervisión eficaz, libros transparentes y precisos, trato justo entre las partes.

Cabe resaltar, la relación que existe entre la Teoría de Agencia y la Gobernanza Corporativa. La Teoría de Agencia plantea y expone los problemas dentro de la organización, mientras la Gobernanza Corporativa plantea las soluciones para evitar los problemas de la Teoría de Agencia.

\section{Gobernanza Corporativa.}

Shleifer y Vishny (1996)" (p. 1, traducido). Langebaek y Ortiz (2007) "Siguiendo a Charles Oman, Steven Fries y Willem Buiter (2003) se puede definir el gobierno corporativo de una empresa como el conjunto de prácticas formales e informales que regulan la relación entre administradores e inversionistas y entre los diferentes tipos de socios o accionistas" (p. 3, pie de página 4) Citado por (Escobar, y Benavides, y Perafán, H 2016, p.218)

La Gobernanza Corporativa es una teoría complementaria a la Teoría de Agencia. Mientras la Teoría de agencia habla de la problemática en las organizaciones generada por el Riesgo Moral y la Selección Adversa a causa de la Asimetría de la información, el Gobierno Corporativo es la teoría que hace frente a estas problemáticas.

Según Oliver Hart (1995), el concepto de gobierno corporativo nace de la problemática presentada en la agencia y a los costos de transacción generados por la Selección Adversa, Asimetría de la Información y Riesgos Morales.

La OECD define gobierno corporativo como los medios internos por las cuales las corporaciones son operadas y controladas. La estructura de gobierno corporativo especifica la distribución de derechos y responsabilidades entre los diferentes participantes en la organización — como la junta, los gerentes, los accionistas y otras partes interesadas - y establece las normas y procedimientos para la toma de decisiones (OECD, 2015).

Según Raghuram G. Rajan y Luigi Zingales (1998) la Gobernanza Corporativa está orientada a la creación de mecanismos y restricciones que regulan las relaciones de intercambio empresarial, generadoras de rentas.

Diane K. Denis y John J. McConnell (2003) definen Gobierno Corporativo como el conjunto de mecanismos, institucionales y de mercado, en el cual las decisiones tomadas dentro de la compañía cumplen con los objetivos de todos los grupos de interés organizacionales (propietarios, accionistas, gerentes, empleados, clientes). 
Balcazar, A. Gobernanza corporativa, una propuesta para el mejoramiento en la gestión administrativa...

El modelo de Gobernanza Corporativa, busca a través de mecanismos, controles y reglas garantizar el tratamiento equitativo entre los accionistas (para evitar la problemática de Selección Adversa), revelar la información y los datos de forma transparente e igualitaria (para evitar Asimetrías en la Información), establece responsabilidades claras para la junta directiva y consejo organizacional, con el fin de lograr la convergencia de intereses y objetivos entre propietarios y gerentes, minimizando de esta forma el Riesgo Moral.

A partir de la década de 1990, se promueve la aplicación en muchas instituciones del denominado "new managerialism", que acentúa aspectos relacionados con indicadores, la competitividad, la eficiencia y los servicios para los usuarios, los que tienen un impacto innegable en la gestión de las organizaciones, tanto del sector público. (Farrell y Morris, 2003; O’Reilly y Reed, 2010; Lynch, Grummell y Devine, 2012; Lynch, 2012). El new managerialism es un sistema de gobernanza de las organizaciones que favorece el control centralizado de la gerencia y posibilita la descentralización de las responsabilidades. Citado por (Ganga, y Ramos, y Leal 2015, p.13).

De acuerdo a la teoría, las problemáticas de la Teoría de Agencia se presentan en todas las organizaciones en las cuales los propietarios delegan la administración a una tercera persona, generando costos adicionales, que comprometen la estructura financiera de la organización, cuya solución es a través de la implementación de la Gobernanza Corporativa.

Para Grant y McGhee (2014) El GC es una composición de mecanismos que buscan hacer frente a los problemas de agencia entre principal y gestor y entre accionistas mayoritarios y minoritarios para garantizar que los proveedores de capital obtengan el respectivo retorno a la inversión. Con esto, un buen GC se traduce en más ganancias o mayor valoración de la empresa. Dicho rendimiento empresarial se puede observar en tres aspectos: i) rentabilidad (ROA o ROE); ii) valor de mercado ( $Q$ de Tobin) o iii) retorno a la inversión (cambio en el precio de las acciones). La implementación de buenos mecanismos de gobernanza también puede mejorar el rendimiento operativo de la siguiente manera: i) inversiones más eficientes, dada la mejora en la supervisión de las operaciones; ii) utilización de recursos de forma más eficiente, dado que se reducen prebendas por la gestión; iii) reducción de las posibilidades de fraude, liquidación de activos u otras formas de desviación de activos de la empresa o flujos de efectivo de los accionistas; iv) disminución del retorno a la inversión, dada la mejor protección a los accionistas y la disminución del riesgo de perder su inversión, esto a su vez se traduce en mayores ingresos; v) mejora en la disponibilidad de financiación externa. Grant y McGhee (2014) citrado por Jara (2014, p. 3-5).

\section{Metodología}

El objetivo de esta investigación es verificar que los problemas planteados por la Teoría de Agencia (Selección Adversa, Riesgos Morales, Asimetrías de la Información), se presentan en la gestión del Hospital de Tabio y que la implementación de los postulados de la Gobernanza Corporativa, los cuales se 
rigen en Colombia a través del Código País, contribuiría a mejorar la gestión administrativa y financiera dentro del Hospital de Tabio (CundinamarcaColombia). Para alcanzar dicho fin se procedió a efectuar una revisión de documentos y artículos, con el fin de demostrar que es viable generar una propuesta de mejoramiento administrativa y financiera, de acuerdo a lo planteado por la Teoría de Agencia y la Teoría de Gobernanza Corporativa.

La revisión bibliográfica es una metodología a implementar en cualquier trabajo de investigación, con el fin de verificar la originalidad y relevancia del tema a investigar.

De acuerdo con esta metodología se desarrollaron tres fases, en la primera se definió el problema de investigación, con el fin de efectuar la respectiva búsqueda bibliográfica. En la segunda fase se consultaron las bases de datos con el de demostrar la pertinencia de la hipótesis formulada en concordancia con el problema planteado. En la tercera fase se analizó la información organizada, para identificar la propuesta a presentar.

De acuerdo con lo anterior el problema de la investigación radica en demostrar que el Hospital Nuestra Señora del Carmen presenta los problemas de Riesgo Moral y Selección Adversa propuestos por la Teoría de Agencia y evidenciar que es posible mejorar la gestión administrativa y financiera dentro del Hospital de Tabio (Cundinamarca- Colombia), aplicando los postulados de la Gobernanza Corporativa, los cuales están enmarcados a través del Código País en Colombia.

De acuerdo con este planteamiento se procede a buscar en los informes de auditoría emitidos por la Contraloría de Cundinamarca, y en las bases de datos: Proquest, Ebsco, Scielo artículos relacionados con la aplicación de la teoría de Gobernanza Corporativa en diferentes escenarios corporativos, resaltando las ideas más relevantes.

Se procede a organizar la información encontrada y se verifica la proximidad de cada uno de los artículos de acuerdo a la hipótesis planteada, en cuadros comparativos, y finalmente se plantea la propuesta de mejoramiento de acuerdo a la información recolectada y analizada.

Con relación a la muestra se escoge al hospital de Tabio para el estudio de casos, teniendo en cuenta que de los hospitales dentro de esta categoría (E.S.E) en Cundinamarca, es uno de los que más población atiende y dentro de los informes verificados, presenta una gestión administrativa y contable desfavorable y no fenece su cuenta. 


\title{
V. Resultados
}

\section{V.1 Gestión administrativa y financiera en el Hospital E.S.E Nuestra Señora del Carmen de Tabio.}

\author{
Tabla 1. Hallazgos de auditoria E.S.E Nuestra Señora del Carmen de Tabio \\ informe de auditoría presentado en el 2018 vigencia 2019, planes de \\ mejoramiento vigencia 2015, 2016, 2017.
}

\begin{tabular}{ll}
\hline \multicolumn{1}{c}{ Concepto } & \multicolumn{1}{c}{ Hallazgo } \\
\hline Evaluación & El Plan de Mejoramiento presentado por la Administración de la E.S.E. Hospital Nuestra \\
plan de & Señora del Carmen de Tabio, como resultado de la Auditoría Gubernamental con Enfoque \\
mejoramiento & Integral Modalidad Integral, efectuada a la vigencia 2013, presentó un avance del 32\%, \\
& lo cual es inaceptable el nivel de cumplimiento, de conformidad con lo previsto en la \\
& Resolución 0150 de 2006 y la Resolución No. 0086 de 04 de febrero de 2010, emitida por \\
& la Contraloría de Cundinamarca. \\
& Se concluye que el Plan de Mejoramiento presentado por la E.S.E., Hospital Nuestra \\
& Señora del Carmen de Tabio, de la Auditoría Gubernamental con Enfoque Integral \\
& Modalidad Integral, correspondiente a la vigencia fiscal 2013, presenta un cumplimiento \\
& del $32 \%$, para las 22 acciones de mejora propuestas a los 11 hallazgos. \\
& No cumple con las acciones correctivas correspondientes. (Informe de Auditoria 2017, \\
p.3)
\end{tabular}

Rendición de La administración del sujeto de control no acató los lineamientos de la Resolución 097 de cuentas 29 de enero de 2016, emitida por la Contraloría de Cundinamarca, respecto a la rendición de la cuenta anual, vigencia 2016, por cuanto se presentó un error en el diligenciamiento del formato F08 al presentarse en el gasto una diferencia de $\$ 39.142 .210$ por un error de transcripción. Adicionalmente en el formato F99 no presentaron el anexo de Indicadores de Gestión.

No fenece la Cuenta.

Archivos No cuentan con lo estipulado en la ley general de archivos, los expedientes no cumplen con el orden cronológico.

Gestión Los hallazgos presentados inciden de manera significativa en los resultados de la administrativa administración, lo que nos permite conceptuar que la gestión adelantada por la Empresa Social del Estado, alcanzó la ejecución del plan de mejoramiento de la auditoría de la vigencia 2013 en $32 \%$. El plan de acción no se cumplió, la encuesta del sistema de control interno reportó una calificación de una valoración total de 33\%, el cual presenta debilidades significativas en el cumplimiento de los criterios de eficiencia y eficacia. Por tal razón el concepto sobre la gestión y resultados es DESFAVORABLE. (Informe de auditoría, 2017, p.11)

Control Las debilidades expresadas anteriormente, obedecen a que la E.S.E. no ha implementado interno los puntos de control evidenciándose el incumplimiento a los plazos y lineamientos establecidos en el Decreto 943 de 2014 para la implementación del MECí.

No cuentan con mecanismos de control y de seguimiento.

Liquidación de Ocho (8) convenios se encuentran sin liquidar desde el año 2013 y suman \$2.167.692.412. convenios 


\begin{tabular}{ll}
\hline \multicolumn{1}{c}{ Concepto } & \multicolumn{1}{c}{ Hallazgo } \\
\hline Estados & La E.S.E. presenta un alto riesgo respecto a la recuperación de cartera, ya que el $46 \%$ del \\
contables & total de la cartera vencida, presenta un vencimiento superior establecido en el rango de \\
& 90 a 180 días equivalentes a \$ 1.055.145.208. \\
& En el pasivo se evidenció una clara identificación de la falta de depuración contable, \\
& se señalan los casos o tareas pendientes de identificar el destino o procedencia de la \\
& información que alimenta los estados contables. Esto conlleva a afectar claramente \\
& el objetivo de la información con el fin de que la alta dirección tome las decisiones \\
& oportunas. \\
& A la fecha de la ejecución del proceso auditor no se evidencia ningún tipo de avance \\
& y/o actuación en pro de desarrollar actividades preventivas en la información financiera, \\
& económica y social de la E.S.E. con el fin de que garanticen la producción de una \\
& información razonable y oportuna. (Informe de auditoría, 2017, p.17) \\
& No existen los manuales de procesos para glosas. \\
& En los estados contables, la glosa se registra en la cuenta contable 83, cuenta de orden \\
Gloudoras de control por \$226.482.456 mientras que los datos reportados en el informe & \\
& que presentó el área de facturación fue de \$139.284.529, observándose una diferencia \\
de \$87.197.927, por lo que corroboramos que no hay evidencia del manejo de la glosa \\
definitiva, ésta es enviada al gasto, cuando ha sido aceptada, pero no se especifica el \\
valor de la misma, ni hay notas contables que expliquen la causa de ésta, aumentando en \\
forma no razonable los gastos. El Informe de auditoría, 2017, p.86)
\end{tabular}

Almacén Según la visita realizada al almacén, el día 24 de febrero del 2017, en la cual el grupo auditor confrontó el ingreso de los elementos adquiridos por la E.S.E., se observan falencias en la funcionalidad del módulo denominado Citisalud, toda vez que al momento de realizarse el seguimiento a los elementos se evidenció que no se lleva un adecuado control al stock, corno tampoco a las salidas de estos, pues el módulo no permite identificar el inventario real que posee el almacén. (Informe de auditoría, 2017, p.95)

Propiedad, No se evidencia documento ni soporte que refleje las actualizaciones de las valorizaciones planta y de esta cuenta.

equipo La E.S.E. No posee los procesos de identificación de todos y cada uno de los activos de la entidad y la actualización de activos fijos (Informe de auditoría, 2017, p.88)

Sanciones La E.S.E. tenía la obligación de realizar el reporte de los precios de compra y/o venta de medicamentos al Sistema de Información de Precios, en lo correspondiente al segundo, tercer y cuarto trimestre de 2011, en las fechas establecidas en las normas citadas y no lo efectúo, lo que origina una sanción.

La E.S.E. en el procedimiento de rendición de informes a otras entidades, no realizó un control y seguimiento adecuado, generando con este incumplimiento una sanción (multa) impuesta por la Superintendencia de Industria y Comercio.

Por lo anterior, se evidencia que la gestión fiscal fue inadecuada, con la imposición de la multa por \$9.595.488, el gestor fiscal no cuidó, ni conservó, correctamente los recursos públicos y se ocasionó un menoscabo al patrimonio de la E.S.E. (Informe de auditoría, 2017, p.59-60)

Talento Se evidencia contratación bajo la modalidad de prestación de servicios, carecen de humano planes de incentivos, no se evidencia control y supervisión de estos contratos por parte de la oficina de control interno.

Fuente. Elaboración propia tomado del informe de Auditoría vigencia 2017, E.S.E Nuestra Señora del Carmen de Tabio emitido por la Contraloría de Cundinamarca. 
Balcazar, A. Gobernanza corporativa, una propuesta para el mejoramiento en la gestión administrativa...

Tabla 2. Problemas planteados por la Teoría de Agencia versus hallazgos del Hospital de Tabio

\begin{tabular}{|c|c|}
\hline Problema & Hallazgo \\
\hline an & $\begin{array}{l}\text { Ante el incumplimiento de las acciones correctivas propuestas se incurre en el problema } \\
\text { de Riesgo Moral, por falta de gestión del encargado del proceso, lo cual evidencia la } \\
\text { discrepancia entre el principal (Estado) y el agente (directivo encargado). }\end{array}$ \\
\hline Rendición de & $\begin{array}{l}\text { Ante la discrepancia de lo reportado en los formatos y el sistema, se observa que se } \\
\text { presenta el problema de asimetrías de información, lo cual provoca el problema de } \\
\text { Selección Adversa dentro del hospital. }\end{array}$ \\
\hline rchivo & $\begin{array}{l}\text { No cuentan con lo estipulado en la ley general de archivos, los expedientes no cumplen } \\
\text { con el orden cronológico, provocando problemas de Asimetría de la Información y } \\
\text { Selección Adversa. }\end{array}$ \\
\hline $\begin{array}{l}\text { Gestión } \\
\text { administrativa }\end{array}$ & $\begin{array}{l}\text { El Hospital genera detrimento al fisco por su gestión desfavorable, lo que evidencia } \\
\text { diferencias de intereses entre el principal y el gestor, se evidencia en consecuencia el } \\
\text { problema de Riesgo Moral. }\end{array}$ \\
\hline Control interno & $\begin{array}{l}\text { Al no contar con mecanismos de control y de seguimiento, se incurre en el problema de } \\
\text { Riesgo Moral ya que el agente no tiene definido las metas a cumplir. Además, Asimetría } \\
\text { de la Información ya que el principal no puede verificar la gestión del agente. También } \\
\text { Problemas de Selección Adversa entre el agente y el principal, en donde el principal no } \\
\text { tiene un referente de medición, lo cual puede favorecer al agente ante su ineficiente } \\
\text { actuación administrativa. }\end{array}$ \\
\hline $\begin{array}{l}\text { Liquidación de } \\
\text { convenios }\end{array}$ & $\begin{array}{l}\text { Ocho (8) convenios se encuentran sin liquidar desde el año } 2013 \text { y suman } \\
\$ 2.167 .692 .412 \text {. Se puede verificar la falta de inoperancia administrativa del agente } \\
\text { (directivo encargado) en detrimento del patrimonio del principal (Estado), lo cual } \\
\text { evidencia el Problema de Riesgo Moral. }\end{array}$ \\
\hline i & $\begin{array}{l}\text { La E.S.E. presenta un alto riesgo respecto a la recuperación de cartera, ya que el } 46 \% \\
\text { del total de la cartera vencida, presenta un vencimiento superior establecido en el } \\
\text { rango de } 90 \text { a } 180 \text { días equivalentes a } \$ 1.055 .145 .208 \text {. Se puede verificar la falta } \\
\text { de inoperancia administrativa del agente (directivo encargado) en detrimento del } \\
\text { patrimonio del principal (Estado), lo cual evidencia el Problema de Riesgo Moral. } \\
\text { A la fecha de la ejecución del proceso auditor no se evidencia ningún tipo de avance y/o } \\
\text { actuación en pro de desarrollar actividades preventivas en la información financiera, } \\
\text { económica y social de la E.S.E. con el fin de que garanticen la producción de una } \\
\text { información razonable y oportuna. (Informe de auditoría, 2017, p.17), se observa que } \\
\text { se presenta el problema de Asimetría de Información, lo cual provoca el problema de } \\
\text { Selección Adversa dentro del hospital. }\end{array}$ \\
\hline Glosas & $\begin{array}{l}\text { No existen los manuales de procesos para glosas. } \\
\text { En los estados contables, la glosa se registra en la cuenta contable } 83 \text {, cuenta de } \\
\text { orden deudoras de control por } \$ 226.482 .456 \text {, mientras que los datos reportados en } \\
\text { el informe que presentó el área de facturación fueron de } \$ 139.284 .529 \text {, observándose } \\
\text { una diferencia de } \$ 87.197 .927 \text {. (Informe de auditoría, } 2017, \text { p.86), En este aspecto se } \\
\text { incurre en el problema de Asimetría de la información. }\end{array}$ \\
\hline
\end{tabular}
incurre en el problema de Asimetría de la información. 
Almacén En este aspecto se verifican que no se llevan control sobre los inventarios, lo cual evidencia el problema de riesgo moral por la falta de gestión administrativa en lo relacionado con el control y el seguimiento. Además, Asimetría de la información ya que no concuerda lo físico con lo reportado en el aplicativo del hospital, lo que incide en el problema de Selección Adversa por estos sesgos en la Información.

Propiedad, planta No se evidencia documento ni soporte que refleje las actualizaciones de las y equipo valorizaciones de esta cuenta. Aquí se puede verificar problemas de Asimetría de la Información y Selección Adversa entre agente y principal.

Sanciones Estas sanciones se generan por inoperancia administrativa (Riesgo Moral), lo que conlleva a un detrimento patrimonial en el Estado.

Talento humano Se evidencia contratación bajo la modalidad de prestación de servicios, carecen de planes de incentivos, no se evidencia control y supervisión de estos contratos por parte de la oficina de control interno. En este aspecto se puede evidenciar que es necesario generar una propuesta en cuanto a incentivos y compensaciones para el personal del Hospital, con el fin de subsanar los problemas y costos de transacción que surgen por el problema de Riesgo Moral.

Lo anterior se puede desarrollar de acuerdo con el Plan de Incentivos y de Estímulos enmarcado en la Ley No. 909 de 2004, reglamentado por el Decreto Ley No. 1567 de 1998 y Decreto No. 1227 de 2005 Artículo 69 al 85.

Fuente. Elaboración propia.

\section{V.2 Gobernanza Corporativa frente al mejoramiento de la gestión administrativa y financiera.}

A continuación, se relacionan los estudios encontrados en los cuales se puede evidenciar el mejoramiento de la gestión administrativa y financiera tras la implementación de propuestas basadas en los postulados de Gobernanza Corporativa.

Tabla 3. Evidencia de la relación directa de la aplicación de la Teoría de Gobernanza Corporativa y el mejoramiento de los indicadores administrativos y financieros dentro de las organizaciones. Recopilación de estudios realizados.

\begin{tabular}{ll}
\hline \multicolumn{1}{c}{ Autores } & \multicolumn{1}{c}{ Posición } \\
\hline Fernando Lefort (2003). & $\begin{array}{l}\text { Lefort argumenta que el Gobierno Corporativo } \\
\text { contribuye a disminuir los costos de un mal } \\
\text { sistema de incentivos o mala asignación de } \\
\text { capital frente a los conglomerados. }\end{array}$
\end{tabular}

Lawrence C. Brown y Marcus L. Caylor (2004a). Este Esta investigación concluye con el desarrollo estudio tiene como propósito elaborar un índice de GC y del índice de medición, que logró establecer la observar la relación con algunas variables de desempeño relación directa del GC frente a las categorías financiero clasificadas en tres categorías: i) desempeño financieras planteadas. operativo (rentabilidad sobre recursos propios, margen de beneficio y crecimiento de las ventas); ii) pago a los accionistas (rentabilidad por dividendo y recompra de acciones); y iii) valoración de la firma ( $\mathrm{Q}$ de Tobin). 
Balcazar, A. Gobernanza corporativa, una propuesta para el mejoramiento en la gestión administrativa...

\section{Autores}

Posición

Ricardo P. C. Leal y André L. Carvalhal da Silva (2004). Concluyen que un buen GC está positivamente Estudio efectuado en las empresas públicas del Brasil, correlacionado con un mayor valor de las que aplican los postulados de la Gobernanza Corporativa. corporaciones en Brasil.

Julián Benavides-Franco (2005) estudió la concentración La conclusión es que existe un efecto positivo de la propiedad y el desempeño contable en un panel de Gobierno Corporativo frente al desempeño de datos de 532 empresas entre 1999 y 2003 de cinco financiero.

países latinoamericanos: Colombia, Brasil, Chile, Perú y Venezuela.

Andrés Langebaek y Jaime Eduardo Ortiz (2007) estudiaron el estado de las prácticas de GC en las empresas que negocian acciones ordinarias en la Bolsa de Colombia, para lo cual construyeron su propio índice: el Índice de Gobierno Corporativo, IGC. Adicionalmente, midieron el impacto que las buenas prácticas de GC tienen sobre los costos de financiación del capital de las empresas. La muestra utilizada es de 57 empresas que negociaban acciones ordinarias en la Bolsa de Colombia en 2005.

Sanjai Bhagat y Brian Bolton (2008).

Existe una relación simultánea entre el desempeño financiero y el Gobierno Corporativo, y lo comprueba con ecuaciones matemáticas.

Por último, el artículo de Julián Benavides Franco y Permite incorporar inequívocamente la relación Samuel Mongrut-Montalván (2010) tiene como objetivo observar los efectos sobre el desempeño contable y las decisiones financieras en empresas colombianas que implementar el código de gobierno (Código País es el nombre de la reglamentación del Gobierno Corporativo para Colombia).

causal en GC y desempeño (o apalancamiento).

La muestra de este estudio es de 43 empresas entre 1997 y 2006. Entre los resultados más importantes de este estudio, están el aumento significativo del ROA después de implementar el código de GC: su efecto fue mayor entre mejor fuera la calidad del código. Así mismo, el endeudamiento de las empresas sube después de emitir el código y cuanto mejor sea su calidad. La conclusión general es que la autorregulación es eficaz como medio para inducir un compromiso de los accionistas controlantes para reducir sus rentas. Además, que los esfuerzos para mejorar las prácticas de GC se traducen en finanzas sólidas.

Jackie Krafft, Yiping Qu, Francesco Quatraro y Jacques- Al confirmar la asociación entre altos niveles Laurent Ravix (2014) de GC y desempeño financiero, hallaron que los retornos accionarios, el rendimiento de los dividendos, el ROA y el margen de utilidad neta responden positivamente a mejoras en los índices de gobierno, lo mismo que la $\mathrm{Q}$ de Tobin para un panel de firmas en 24 países y sectores industriales entre 2003 y 2008.

Fuente. Elaboración propia. Tomado de Escobar, y Benavides, y Perafán, H 2016, p.230-237. 


\section{V.3 Propuesta implementación del Código País (Gobernanza Corporativa) Hospital E.S.E Nuestra Señora del Carmen Tabio}

Teniendo en cuenta la revisión efectuada cuyos resultados se encuentran en el cuadro anterior, se puede observar que, al aplicar las ideas de Gobernanza Corporativa, para minimizar la Selección Adversa y el Riesgo Moral, se incide significativamente en el mejoramiento administrativo y financiero de las organizaciones.

En consecuencia, es pertinente proponer a la gerencia del hospital de Tabio la implementación del modelo de Gobernanza corporativa (Código País), con el fin de mejorar su gestión administrativa y financiera minimizando los problemas de Riesgo Moral, Selección Adversa y Asimetrías de la Información.

De acuerdo al Informe pormenorizado del estado de control interno (2017) Hospital E.S.E Nuestra Señora del Carmen Tabio, se evidencian problemas de Selección Adversa y de Riesgo Moral.

De acuerdo a este informe el Hospital de Tabio no cuenta con un personal comprometido con la institución (Riesgo Moral), el personal está contratado bajo la modalidad de prestación de servicios, se presenta alta rotación de personal, mal clima laboral, carece de un proceso de control establecido, existe un órgano auditor pero no poseen un manual de auditoria, existen dificultades en el seguimiento a los indicadores de gestión y financieros, carecen de un sistema de control y verificación de inventarios, no existe un plan de compras con necesidades definidas, carecen de medios y estrategias de difusión de la información (Problemas de Asimetría de la Información y en consecuencia Selección Adversa).

De acuerdo a las disposiciones del Código País, se deben plantear medidas concretas con relación a la Asamblea de Accionistas, Junta Directiva, revelación de la información Financiera y no financiera y solución de controversias. Estas medidas concretas deberán ser supervisadas por el comité de Gobernanza Corporativa. Cabe anotar que, en el caso particular del Hospital de Tabio, este no cuenta con Asamblea de Accionistas, es en cabeza del departamento de Cundinamarca y del municipio que recae esta figura.

Se sugiere en consecuencia la creación de un Comité de Gobernanza Corporativa como órgano independiente, que se encargue de la verificación de la gestión del Gerente y Directivos del hospital, el cual deberá contar con procesos claros de seguimiento, deberán establecer una pila de indicadores medibles en períodos cortos de tiempo, con el fin de determinar el cumplimiento de metas dentro del Hospital. Este Comité debe ser apoyado por el Comité de Auditoría, por lo cual es necesario crear manuales de procesos y procedimientos claros para las diferentes áreas del Hospital para mejorar el control y divulgación de la información de la organización. 
El Comité deberá sugerir que la Junta Directiva del Hospital de Tabio, se encargue de efectuar planes estratégicos, establecer necesidades de compra de acuerdo a las necesidades reales de la entidad; en este aspecto se deben generar los controles respectivos, se debe incentivar a los directivos (reconocimientos a favor de la reputación de los gestores) por el cumplimiento de metas del Hospital y de esta forma evitar los Riesgos Morales (toma de decisiones, planes y procesos para beneficios particulares, no para beneficio de la institución).

El Comité deberá proponer una política de salarios y remuneración, de igual forma un plan de compensación para los miembros de la organización, en especial la alta gerencia; evitando de esta forma que directivos del hospital utilicen a la organización como un trampolín político.

El Comité debe establecer claramente objetivos y protocolos de contratación y efectuar estos de acuerdo a las necesidades reales del Hospital, sin intervenciones de tipo burocrático. De igual forma se debe verificar las manuales funciones y los perfiles del cargo acorde a las funciones establecidas y divulgación respectiva de las hojas de vida.

El Comité deberá estipular los marcos regulatorios en el Hospital de acuerdo a la reglamentación nacional estipulada en: artículos 42 de la Ley 715 de 2001 y 17 de la Ley 812 de 42 de la Ley 715 de 2001 (normatividad para sistemas de evaluación), artículo 43 de la Ley 715 de 2001 (normatividad para el reporte de información del sector), artículo 17 de la Ley 812 de 2003 (normatividad para la gestión pública), artículo 52 de la Ley 812 de 2003 y decreto 2193 del 2014 Artículo $1^{\circ}$ (normatividad que estipula como se debe entregar la información del sector), Conpes 3204 del 6 de noviembre de 2002 (normatividad para el plan de inversión). 
Tabla 4. Elementos y criterios de la propuesta de Gobernanza Corporativa para el Hospital E.S.E Nuestra Señora del Carmen Tabio, de acuerdo a las disposiciones del Código País.

\begin{tabular}{lll}
\hline \multicolumn{1}{c}{ Elementos } & \multicolumn{1}{c}{ Criterios } & \multicolumn{1}{c}{ Actividades a desarrollar } \\
\hline Comité de & Órgano independiente & -Crear el manual de procesos y procedimientos \\
Gobernanza & encargado de la verificación & para la toma de decisiones de la Junta Directiva. \\
Corporativa. & de gestión del Gerente y & -Crear el manual de procesos y procedimientos \\
& Directivos del hospital, deberá & para la divulgación de información financiera y no \\
contar con procesos claros de & financiera. \\
& seguimiento, deberá establecer & -Crear el manual de procesos y procedimientos \\
una pila de indicadores & para la resolución de conflictos al interior de la \\
medibles en períodos cortos & organización. \\
de tiempo, con el fin de & -Crear indicadores de gestión para la junta directiva \\
determinar el cumplimiento de & en control del cumplimiento de las metas del \\
metas dentro del hospital. Este & Hospital, manejo y divulgación de la información. \\
comité debe ser apoyado por el & -Crear los manuales de procesos y procedimientos \\
comité de auditoría. & para las compras de la entidad. \\
& -Crear la política de incentivos y reconocimientos \\
& a los directivos de acuerdo a las metas cumplidas. \\
& -Proponer una política de salarios y remuneración, \\
& de igual forma un plan de compensación para los \\
& miembros de la organización, \\
& Controly seguimiento a la Asamblea de Accionistas, \\
& Junta directiva, a los procesos de revelación de \\
& la información financiera y no financiera y de \\
& solución de controversias.
\end{tabular}

Representantes de la

Gobernación de Cundinamarca (equivalente a la Asamblea General).

Junta Directiva
Participación de los En este aspecto el comité de Gobernanza representantes de la alcaldía y Corporativa deberá:

la gobernación en las reuniones -Crear el proceso de convocatoria para los de la junta directiva. representantes de la alcaldía y gobernación.

-Crear informes de los temas a tratar por la junta directiva, los cuales deben darse a conocer a los representantes de la alcaldía y la gobernación con anterioridad a las reuniones respectivas.

Periodicidad de las reuniones de En este aspecto el comité de Gobernanza la Junta Directiva, verificación Corporativa deberá:

de indicadores de gestión -Crear el reglamento interno de la Junta Directiva. de los avances de los planes -Establecer los planes estratégicos y metas a seguir estratégicos establecidos por la por la Junta Directiva. Junta Directiva y sus metas. Verificación de los indicadores financieros y cumplimientos de compra del hospital. 
Balcazar, A. Gobernanza corporativa, una propuesta para el mejoramiento en la gestión administrativa...

\begin{tabular}{|c|c|c|}
\hline Elementos & Criterios & Actividades a desarrollar \\
\hline $\begin{array}{l}\text { Manejo de la } \\
\text { información } \\
\text { financiera y no } \\
\text { financiera }\end{array}$ & $\begin{array}{l}\text { Nivel de divulgación de la } \\
\text { información. }\end{array}$ & $\begin{array}{l}\text { En este aspecto el comité de Gobernanza } \\
\text { Corporativa deberá: } \\
\text {-Promover el desarrollo de una página web en } \\
\text { la entidad en la cual se carguen las actas de } \\
\text { las reuniones por parte de la junta directiva, } \\
\text { la estructura organizacional, los manuales de } \\
\text { procesos y procedimientos de la entidad, los } \\
\text { estados financieros y contables. }\end{array}$ \\
\hline $\begin{array}{l}\text { Resolución de } \\
\text { controversias }\end{array}$ & $\begin{array}{l}\text { Nivel de problemáticas dentro } \\
\text { de la organización }\end{array}$ & $\begin{array}{l}\text { En este aspecto el comité de Gobernanza } \\
\text { Corporativa deberá: } \\
\text {-Crear un manual de procesos y procedimientos } \\
\text { para la resolución de conflictos. }\end{array}$ \\
\hline
\end{tabular}

Fuente. Elaboración propia.

\section{VI.Conclusiones}

La Gobernanza Corporativa y Teoría de Agencia, no son aplicadas solamente a organizaciones de tipo financiero. De acuerdo a los estudios se pueden identificar que las problemáticas planteadas por la Teoría de Agencia se encuentran al interior de la gran mayoría de las organizaciones. En consecuencia, la Gobernanza Corporativa y lo que implica se aplica de igual manera en la gran mayoría de las empresas. La evidencia muestra que existe una relación directa entre la aplicación del modelo de Gobernanza Corporativa y el mejoramiento de los indicadores administrativos y financieros dentro de la organización.

Dentro del Hospital de Tabio se identifican problemáticas inherentes al Riesgo Moral y a procesos de Selección Adversa.

La discusión en consecuencia radica en porque ante la existencia del Código País, el modelo de Gobernanza Corporativa no es implementado alrededor de estas organizaciones, teniendo en cuenta que el objeto social de los hospitales es considerado de tipo social que afecta directamente a una de las necesidades vitales de la población como es la salud. En consecuencia, es necesario replantear los modelos administrativos implementados en estas organizaciones, con el fin de lograr el mejoramiento administrativo y financiero dentro de ellas, contribuyendo a la población afectada a través de mejores servicios, minimizando costos innecesarios derivados de los problemas de la Teoría de Agencia.

La propuesta presentada es un piloto que podría implementarse en todas las E.S.E de Cundinamarca, incluso del país que presenten situaciones similares a la presentada en el Hospital Nuestra Señora del Carmen del municipio de Tabio, en el cual se implementará la propuesta y se medirán los impactos respectivos. 


\section{Referencias bibliográficas}

Brown, Lawrence D. y Caylor, Marcus L. $(2004<)$. Corporate Governance and Firm Performance. Disponible en: http:// www.whartonwrds.com/wp-content/ uploads/2016/05/Sept-23-CorporateGovernance-and-Firm-Performance.pdf

Bhagat, Sanjai y Bolton, Brian (2008). Corporate Governance and Firm Performance. Journal of Corporate Finance, 14 (3), 257-273. Disponible en: http:// leeds-faculty. colorado.edu/bhagat/ GovernancePerformance-JCF-June2008. pdf, doi:10.1016/j. jcorpfin.2008.03.006

Benavides-Franco, Julián y Mongrut-Montalván, Samuel (2010). Governance Codes: Facts or Fictions? A Study of Governance Codes in Colombia. Estudios Gerenciales, 26 (117), 85-102. Disponible en: http://www. redalyc.org/pdf/212/21218551004.pdf

Benavides-Franco, Julián (2005). Concentración de la propiedad y desempeño contable: el caso latinoamericano. Borradores de Economía y Finanzas, 4, 1-35. Disponible en: https:// www.icesi.edu.co/departamentos/ economia/publicaciones/docs/BORRAECO/ Borrador\%20de\%20Economia\%204.pdf

Claessens, Stijn y Yurtoglu, Burcin (2013). Corporate Governance in Emerging Markets: A Survey. Emerging Market Review, 15 (C), 1-33.

Davis, Jam H.; Schoorman, F. David y Donaldson, Lex (1997). Toward a Stewardship Theory of Management. Academy of Management Journal, 22 (1), 20-47.

Denis, K. y McConnell, John J. (2003) International Corporate Governance. European Corporate Governance Institute, ECGI - Finance Working Paper, 05/2003; Tuck School of Business at Dartmouth Tuck-JQFA Contemporary Corporate Governance Issues II Conference. Disponible en: http://papers.ssrn.com/sol3/ papers.cfm?abstract_ id $=320121$

Donaldson, Lex (1990). The Ethereal Hand: Organizational Economics and Management Theory. Academy of Management Review, 15 (3), 369-381. doi:10.5465/ AMR.1990.4308806

Donaldson, Lex y Davis, James H. (1991). Stewardship Theory or Agency Theory: CEO Governance and Shareholder Returns. Australian Journal of Management, 1 (16), 4964. Disponible en: http://citeseerx.ist. psu.edu/viewdoc/download?doi=10.1.1.199. 6439yrep $=$ rep 1 ytype $=$ pdf

Escobar, N. y Benavides, J y Perafán, H (2016). Gobierno Corporativo y desempeño financiero: Conceptos teóricos y evidencia empírica. doi:10.11144/Javeriana.cc17-43. gcdf. / vol. 17 / no. 43 / enero-junio 2016. http://www.scielo.org.co/pdf/cuco/v17n43/ v17n43a08.pdf

Farrell, C., y Morris, J. (2003). The Neo-Bureaucratic State: Professionals, Managers and Professional Managers in Schools, General Practices and Social Work. OrganizationInterdisciplinary Journal of Organization theory and Society, 10, 129-156.

Ganga, F. y Ramos M, y Leal A (2015). Teoría de Agencia: Supuestos teóricos aplicables. Rev. innovar vol. 25, núm. 57, Julio-Septiembre de 2015. http://www.scielo.org.co/pdf/ inno/v25n57/v25n57a02.pdf. 
Balcazar, A. Gobernanza corporativa, una propuesta para el mejoramiento en la gestión administrativa...

Grant, Patricia y McGhee, Peter (2014). Corporate Governance Reform: Character-Building Structures. Business Ethics: A European Review, 23 (2), 125-138. doi:10.1111/ beer. 12043

Gruszczynski, Marek (2006). Corporate Governance and Financial Performance of Companies in Poland. International Advances in Economic Research, 12 (2), 1-10.

Hart, Oliver (1995). Corporate Governance: Some Theory and Implications. Economic Journal, 105 (430), 678-689. Disponible en: http:/www.ppge.ufrgs.br/giacomo/ arquivos/gov-corp/hart-1995.pdf.

Contraloría de Cundinamarca (2017), Informe de auditoría Hospital E.S.E Nuestra Señora del Carmen. Tabio. http://www.contraloriadecundinamarca.gov.co/index.php/es/2014-1210-21-50-00/proceso-auditor/plan-general-de-auditoria/sector-departamental-2014/ pga-es-co-2

Jensen, M., y Meckling, W. (1976). Theory of the Firm: Managerial Behavior, Agency Costs and Ownership Structure. Journal of Financial Economics, 3(4), 305-360.

Krafft, Jackie; Qu, Yiping; Quatraro, Francesco y Ravix, Jacques-Laurent (2014). Corporate Governance, Value and Performance of Firms: New Empirical Results on Convergence from a Large International Database. Industrial and Corporate Change, Oxford University Press, 23 (2), 361-397.

Langebaek, Andrés y Ortiz, Jaime Eduardo (2007). Q de Tobin y gobierno corporativo de las empresas listadas en bolsa. Borradores de Economía, 447, 1-37. Disponible en: http://www.banrep.gov.co/docum/ftp/ borra447.pdf

Leal, Ricardo P. C. y Carvalhal da Silva, André L. (2005). Corporate Governance and Value in Brazil (and in Chile). Washington: InterAmerican Development Bank, Latin American Research Network, Research Network Working paper R-514. Disponible en http:// www.istfin.eco.usi.ch/r_leal.pdf

Lefort, Fernando (2003). Gobierno corporativo: ¿qué es? y ¿cómo andamos por casa? Cuadernos de Economía, 40 (120), 207-237. Disponible en: http://www.scielo. cl/ scielo.php?script $=$ sci arttextypid $=$ S0717-68212003012000002

Organization for Economic Co-operation and Development, OECD (2015). G20/ OECD Principles of Corporate Governance. Paris, OECD. Disponible en: http://www. oecd. org/corporate/principles-corporategovernance.htm

Oman, Charles; Fries, Steven y Buiter, Willem (2003). Corporate Governance in Developing, Transition and Emerging-Market Economies. Francia: OCDE Development Centre, Policy Brief 23. Disponible en: http://www.oecd. org/dev/poverty/28658158.pdf

Rajan, Raghuram G. y Zingales, Luigi (1998). Financial Dependence and Growth. American Economic Review, 88 (3), 559-586.

Shleifer, Andrei y Vishny, Robert W. (1997). A Survey of Corporate Governance. The Journal of Finance, 52 (2), 737-783. 